\title{
Symmetry Analysis in Metallic Glasses by Electron Nanodiffraction
}

\author{
Shuoyuan Huang, Carter Francis and Paul Voyles
}

University of Wisconsin Madison, United States

Rotational symmetries in electron nanodiffraction (END) measured by 4D STEM can reveal and map local structure in metallic glasses [1][2]. The angular autocorrelation function and its power spectrum have been used to characterize local symmetries, but we show that it can exhibit several types of artifacts. We have adapted the Symmetry STEM method, recently proposed to probe local symmetry in 4D STEM data from crystals [3], to analyze rotational symmetry in 4D STEM data from metallic glasses without artifacts.

Fig. 1(a) - (c) illustrates angular correlation analysis. Individual diffraction patterns $I(\boldsymbol{k})$, are unwrapped into polar coordinates $I(k, \varphi)$ and autocorrelated in azimuth to obtain

$$
C(k, \Delta)=\frac{\langle I(k, \varphi) I(k, \varphi+\Delta)\rangle_{\varphi}-\langle I(k, \varphi)\rangle_{\varphi}^{2}}{\langle I(k, \varphi)\rangle_{\varphi}^{2}}
$$

Rotational symmetry in $I(\boldsymbol{k})$ is transformed in periodic symmetry in $C(k, \Delta) . C(k, \Delta)$ is then Fourier transformed to obtain the power spectrum $P(k, n)$. A simple mathematical model for a general END pattern illustrates three artifacts present in angular correlation analysis. Assume the END pattern consists of $m+1$ pairs of diffraction disks (speckles) all at the $k$ radius, with $m$ of the pairs rotated by angles $\left\{\Delta_{1}, \Delta_{2}, \ldots, \Delta_{m}\right\}$ with regards to a reference pair. The disk intensities are $\left\{p_{0}, p_{0}{ }^{\prime}, p_{1}, p_{1}{ }^{\prime}, \ldots, p_{\mathrm{m}}, p_{\mathrm{m}}{ }^{\prime}\right\}$ where $p_{i}$ and $p_{i}{ }^{\prime}$ are the intensities of the ith pair. The angular power of this pattern at $k$ is given by

$$
P(n)=\frac{\sin ^{2}(\pi n w / N)}{\sin ^{2}(\pi n / N)}\left[\sum_{i}\left(p_{i}+(-1)^{n} p_{i}^{\prime}\right)^{2}+\sum_{i, j, i>j} 2\left(p_{i}+(-1)^{n} p_{i}^{\prime}\right)\left(p_{j}+(-1)^{n} p_{j}^{\prime}\right) \cos q\left(\Delta_{i}-\Delta_{j}\right)\right]
$$

where $N$ is the total number of discrete azimuthal angles, $w$ is the width of each disk in $\varphi$, and $q=2 \pi \mathrm{n} / \mathrm{N}$.

Eq. 1 demonstrates two of the artifacts in $P(n)$. First, the Fourier transformation of the rectangular functions describing the speckles creates ringing that smears 2-fold symmetry power into high order even symmetries as represented by the $\sin ^{2}$ term in Eq. 1. A second artifact arises from the breakdown of Friedel symmetry in the pattern, meaning that $I(\boldsymbol{k}) \neq I(-\boldsymbol{k})$ or, in this model, $p_{i} \neq p_{i}$. Lens aberrations and defocus and dynamical diffraction in thicker samples can all cause Friedel breakdown in amorphous END patterns [4], none of which are of structural origin. Friedel breakdown gives rise to nonstructural odd $n$ magnitude in the power spectrum, transferred from structural even $n$ power. The third common artifact is random arrangements of speckles arising from multiple scatterers separated in the sample thickness. The random inter-cluster overlap as well as intra-cluster structural correlation manifest themself in the angular power as the cosine term in Eq. 1 and act as modulations between pairs. In this analysis, they are practically indistinguishable from one another. Collectively, these three artifacts can make angular correlation results challenging to interpret. 
Fig 1(d) - (e) illustrates our new analysis inspired by Symmetry STEM. It starts by rotating the pattern around its origin by an angle $\Delta$, then calculating the Pearson product-moment correlation coefficient between the original and rotated patterns,

$$
\rho(\Delta)=\frac{\langle I(k, \varphi) I(k, \varphi+\Delta)\rangle_{k, \varphi}-\langle I(k, \varphi)\rangle_{k, \varphi}^{2}}{\left\langle I(k, \varphi)^{2}\right\rangle_{k, \varphi}-\langle I(k, \varphi)\rangle_{k, \varphi}^{2}}
$$

$\rho(\Delta)$ can be averaged over the full range of $k$ in the pattern or a selected range of $k$ values. The symmetry coefficients $S(n)$ are evaluated from $\rho(\Delta)$ following two rules: (1) $S(n)$ is the mean of $\rho(\Delta)$ at the characteristic angles of $n$-fold rotation symmetry, excluding $\Delta=0$ and $2 \pi$; and (2) $S(2 n)$ doesn't sample angles already included in $S(n)$. The first few terms of $S(n)$ are defined as

$$
\begin{aligned}
& S(2)=\rho(\pi) \\
& S(3)=[\rho(2 \pi / 3)+\rho(4 \pi / 3)] / 2 \\
& S(4)=[\rho(\pi / 2)+\rho(3 \pi / 2)] / 2 \\
& S(5)=[\rho(2 \pi / 5)+\rho(4 \pi / 5)+\rho(6 \pi / 5)+\rho(8 \pi / 5)] / 4 \\
& S(6)=[\rho(\pi / 3)+\rho(5 \pi / 3)] / 2
\end{aligned}
$$

The odd $n S(n)$ contain contributions both from inherent odd symmetries in the pattern and from even symmetries that are twice the odd (for instance, $S(3)$ could come from both 3- and 6-fold symmetry). However, $S(6)$ is independent of $S(3)$ by construction.

$S(n)$ avoids the first two artifacts of the angular correlation power spectrum. Without the Fourier transformation, there is no ringing effect, and no nonstructural odd symmetries from Fourier decomposition of uneven intensity speckles due to Friedel symmetry breakdown. Random overlaps still effect $S(n)$, but their influence is reduced. Unlike in $P(n), S(n)$ measures correlations only at the exact angles of the rotational symmetry. Correlations close to but not at those angles will not be registered, which significantly reduces the chance of random overlap creating a high magnitude, even symmetry. However, the odd symmetry coefficients sample more angles than even ones, so odd symmetries are more likely to gain magnitude from random overlaps.

Fig. 2 shows autocorrelation power spectrum and symmetry coefficient analysis of END experiments on $\mathrm{Pd}_{43} \mathrm{Ni}_{10} \mathrm{Cu}_{27} \mathrm{P}_{20}$ nanowires. 960 END patterns are acquired with a parallel probe $2 \mathrm{~nm}$ in diameter from $\sim 40 \mathrm{~nm}$ thick wires. Because the sample is fairly thick, the angular power spectrum in Fig.2a shows a monotonic decrease in magnitude with symmetry order $\mathrm{n}$. Random overlaps and possible dynamical diffraction creates significant Friedel symmetry breakdown, which in turn causes strong nonstructural odd symmetry that overshadows the even symmetry features in the power spectrum. On the other hand, the averaged symmetry coefficient in Fig 2b shows strong 6-fold and 10-fold symmetry, which are characteristic of icosahedral structure. These results suggest that symmetry analysis is more tolerant of thicker samples.

Examining single patterns gives several instances of artifacts in the angular power spectrum which do not influence the symmetry coefficients. Fig. 2c shows a random overlap of speckles at an angle of $\sim 125$ degrees. The power spectrum falsely picks this up as 6-fold symmetry, but the symmetry coefficient does not. Fig. $2 \mathrm{~g}$ shows a 10-fold symmetry that is only partially complete in the diffraction plane. The power 
spectrum recognizes it only as 3-fold symmetry because the three strong speckles are close to the 3-fold positions. The symmetry coefficient registers the 10 -fold angles even though the pattern is incomplete.

We acknowledge support from the NSF (DMR-1807241).
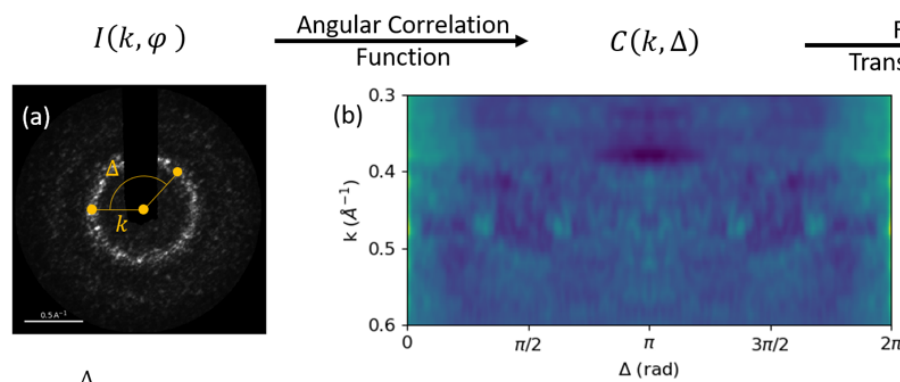

$\stackrel{\text { Fourier }}{\longrightarrow} \hat{C}(k, n)$

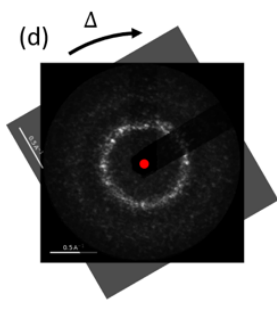

(e)
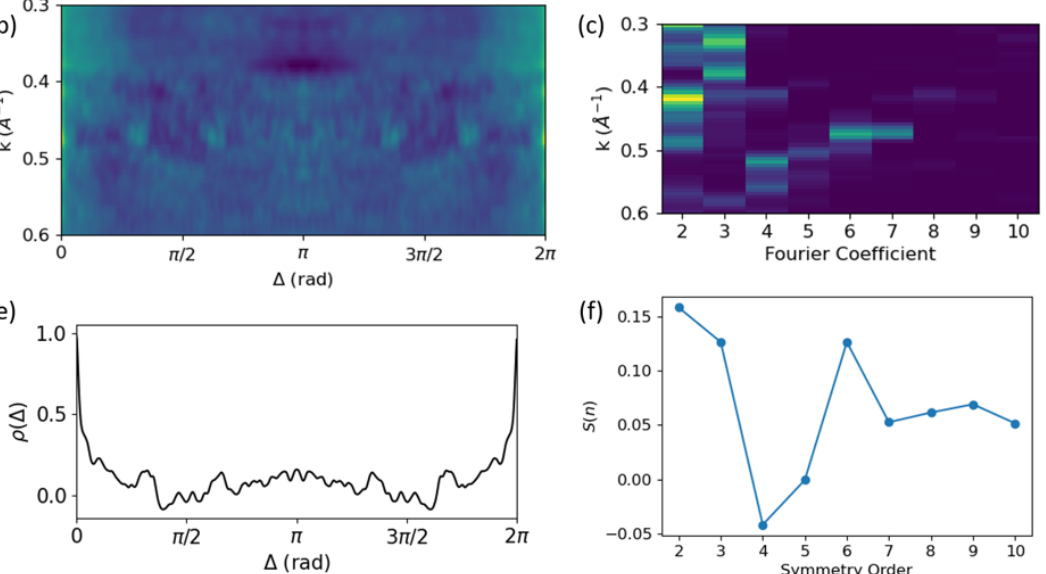

$I(k, \varphi)$

$\underset{\text { Coefficient }}{\stackrel{\text { Pearson Correlation }}{\longrightarrow}}$

$\rho(\Delta)$

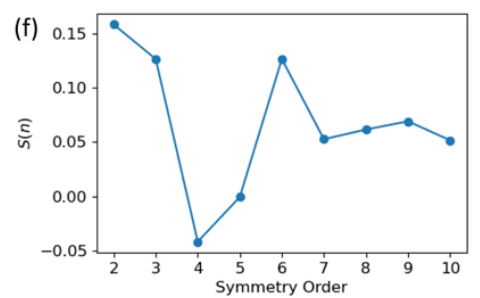

Symmetry

Coefficient

$S(n)$

Figure 1. Schematics of angular correlation analysis and correlation symmetry analysis using an experimental END pattern on Pd-based nanowire sample. (a-c) Angular correlation analysis procedure; (d-f) Symmetry coefficient analysis procedure. 

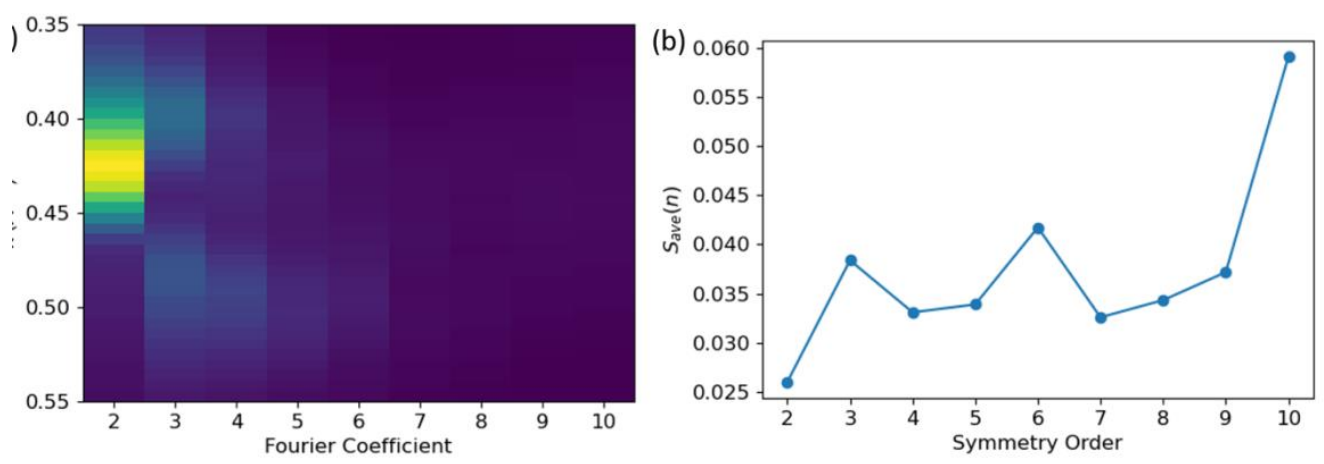

(c)

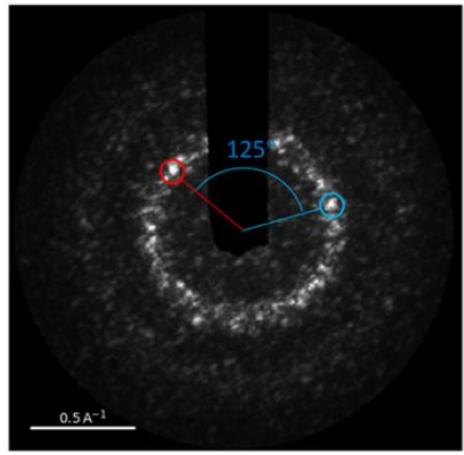

(e)

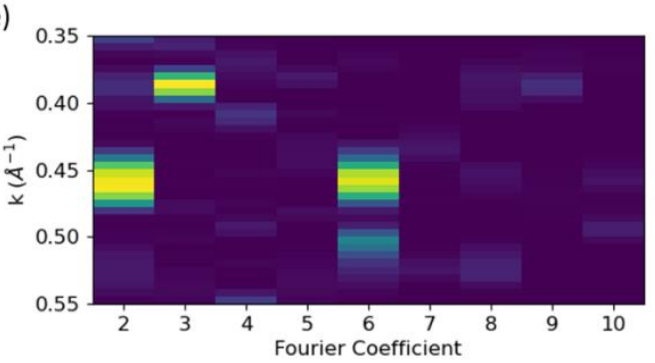

(d)
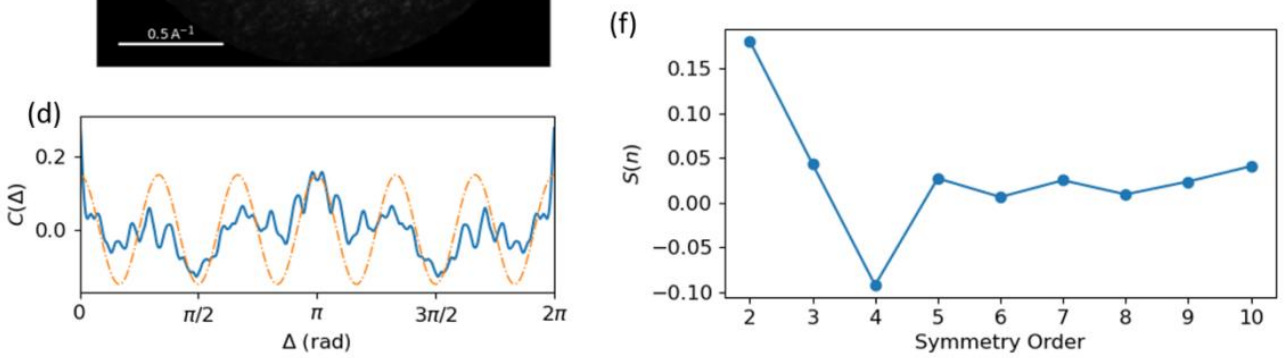

(g)

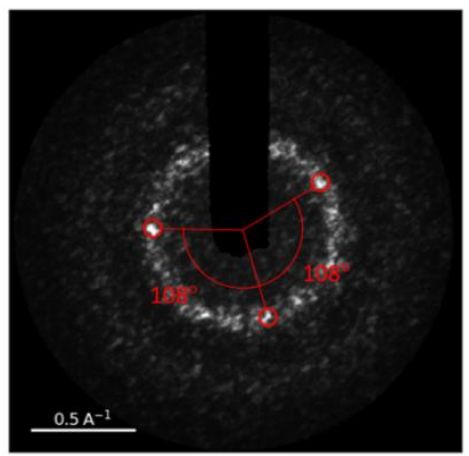

(h)
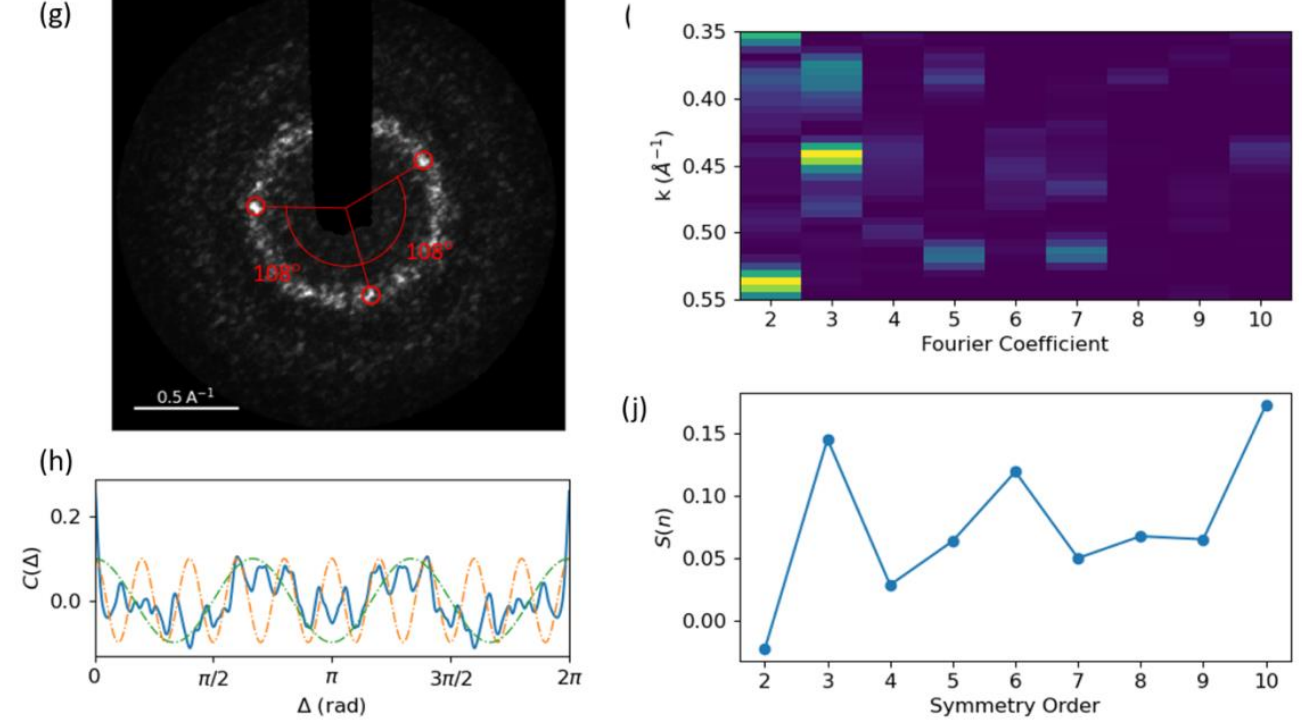

(j)

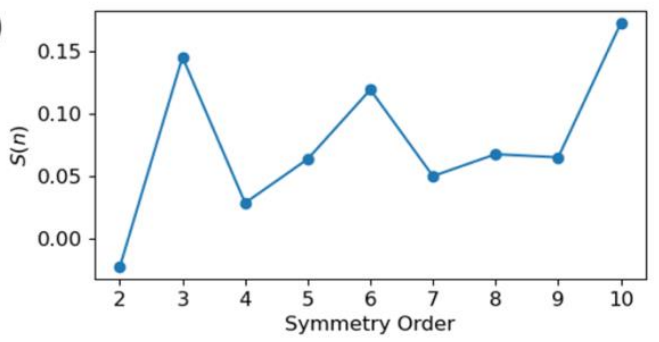

Figure 2. (a) Averaged power spectrum and (b) averaged symmetry coefficients of the END experiments on the Pd-based nanowires; (c, g) examples of END patterns, $(\mathrm{d}, \mathrm{h})$ their angular correlation functions at $\mathrm{k}=\mathrm{kmax},(\mathrm{e}, \mathrm{i})$ power spectrum and (f, j) symmetry coefficients. 


\section{References}

[1] A.C.Y. Liu, M.J. Neish, G. Stokol, G.A. Buckley, L.A. Smillie, M.D. De Jonge, R.T. Ott, M.J. Kramer, L. Bourgeois, Systematic mapping of icosahedral short-range order in a melt-spun $\mathrm{Zr}_{36} \mathrm{Cu}_{64}$ metallic glass, Phys. Rev. Lett. 110 (2013) 1-5.https://doi.org/10.1103/PhysRevLett. 110.205505.

[2] S. Im, Z. Chen, J.M. Johnson, P. Zhao, G.H. Yoo, E.S. Park, Y. Wang, D.A. Muller, J. Hwang, Direct determination of structural heterogeneity in metallic glasses using four-dimensional scanning transmission electron microscopy, Ultramicroscopy. $195 \quad$ (2018) 189-193. https://doi.org/10.1016/j.ultramic.2018.09.005.

[3] M. Krajnak, J. Etheridge, A symmetry-derived mechanism for atomic resolution imaging, Proc. Natl. Acad. Sci. U. S. A. 117 (2020) 27805 27810. https://doi.org/10.1073/pnas.2006975117.

[4] J.M. Rodenburg, Properties of electron microdiffraction patterns from amorphous materials, Ultramicroscopy. 25 (1988) 329-343.

3991(88)90007-1.

https://doi.org/https://doi.org/10.1016/0304- 